\title{
Batch Transfer Integration of RF Microrelays
}

\author{
Veljko Milanović, Michel Maharbiz, and Kristofer S. J. Pister
}

\begin{abstract}
This letter presents the first implementation of batchtransferred microrelays for a broad range of RF applications and substrates. The transferred relays include two types of electrostatic pull-down structures for series and shunt switching of a CPW. The batch-transfer methodology allows integration of optimized microelectromechanical systems (MEMS) in RF systems on substrates such as sapphire, GaAs, and even CMOS. Gold-to-gold contact series microrelays with insertion loss of $<0.3 \mathrm{~dB}$, and isolation better than $15 \mathrm{~dB}$ at frequencies from $100 \mathrm{MHz}$ to $50 \mathrm{GHz}$ are demonstrated, as well as shunt switches with $>45 \mathrm{~dB}$ of isolation and $<0.3$ $\mathrm{dB}$ insertion loss in that frequency range.
\end{abstract}

Index Terms-Batch transfer, flip-chip bonding, microelectromechanical systems, RF MEMs, RF switch.

\section{INTRODUCTION}

$\mathbf{R}$ APID growth of integrated circuit compatible micromachining technologies has spawned extensive research efforts in applications to wireless communications [1], [2]. The developments in microfabrication technology and microelectromechanical systems (MEMS) have made possible the fabrication of actuators suitable for switching microwave signals. Consequently, miniaturization and integration of relays and switches for microwave applications has been the topic of extensive research [2]-[6]. Recent research efforts have been focused into lowering the operating voltages, improving insertion loss and isolation, lowering the cost of fabrication, achieving integration with present technologies, etc.

More recently, there have been efforts in combining the flip-chip packaging technologies and MEMS in RF applications [7], [8]. In batch transfer integration methodology [9], [10], MEMS devices can be fabricated in a custom, optimized process, and transferred onto another substrate after release. The key advantage in RF systems is complete process decoupling-the MEMS structures are fabricated and optimized separately from the RF circuits and transmission lines, allowing integration of optimized MEMS in RF systems on substrates such as sapphire, GaAs, and even CMOS. Another very important advantage of this methodology is that the devices are initially fabricated upside-down before transfer. This allows for the fabrication of elaborate and optimized electromechanical structures before the deposition of relay metals such as gold, or other metals and alloys which are in most cases incompatible with CMOS/MEMS processes. After transfer, these metal contact plates are beneath the MEMS structure and over a target microwave structure to be switched.

We demonstrate for the first time the feasibility of batch transfer integration of RF microrelays. This is demonstrated

Manuscript received January 24, 2000; revised June 6, 2000

The authors are with the Berkeley Sensor and Actuator Center, University of California, Berkeley CA 94720-1770 USA (e-mail: veljko@eecs.berkeley.edu). Publisher Item Identifier S 1051-8207(00)07040-9. with electrostatic pull-down structures with isolated contact plates, actuated similarly as previously proposed switches [5]. Two types of pull-down structures were implemented-series type, where the contact plate connects the discontinued circuit when the structure is down; and the shunt type, where the contact plate shorts the transmission line when down. In both cases, the switches were designed for direct gold to gold contact, instead of capacitive switches [3], which are severely limited at lower frequencies (generally $<10 \mathrm{GHz}$ ). This allows operation down to dc, and well into millimeter-wave frequencies, which is important in instrumentation applications.

\section{FABRICATION OF THE DEVICES}

In order to demonstrate the designed structures and batch transfer integration, we fabricated the MEMS devices on donor wafers, and the microwave structures on target wafers in separate processes. Both process flows, as well as the flip-chip batch transfer, are schematically shown in Fig. 1.

The microwave test structures are all based on coplanar waveguide (CPW) transmission lines, and were fabricated in gold on a quartz target wafer for low loss propagation. These simple test structures and CPW calibration sets were fabricated with a single metal mask. The MEMS devices were fabricated in the following four-mask process [see Fig. 1(a)]. Starting with a p-type $100 \mathrm{~mm}$ silicon donor wafer, $0.6 \mu \mathrm{m}$ of poly is deposited and patterned over $2 \mu \mathrm{m}$ of sacrificial oxide. The poly features act as "pull-down" electrodes. Subsequently, $1 \mu \mathrm{m}$ of low-stress nitride is deposited and patterned as the structural material. This layer of nitride acts as isolation for the poly pull-down plates as well, preventing shorting of the electrostatic plates in the finished relays. A $100 \AA / 5000 \AA \mathrm{Cr} / \mathrm{Au}$ metal layer is subsequently evaporated and patterned as the relay contact plates [see Fig. 1(a)]. Another $150 \AA / 600 \AA \mathrm{Cr} / \mathrm{Au}$ layer is evaporated as a seed layer; 6-8 $\mu \mathrm{m}$ gold bumps are then electroplated in a resist mold. At this point, donor wafers are diced. After resist-stripping, the structures on donor wafers are then released in concentrated in $\mathrm{HF}$ for $\sim 4 \mathrm{~min}$. The released structures remain on the individual chips due to the small gold bump staples [9], which were plated over the structures and surrounding silicon as shown in Fig. 1(b). At that point, the devices are batch-transferred using a flip-chip bonder after careful alignment of donor and target chips, as illustrated in Fig. 1(b). Details of the gold bump transfer process were given previously in [9] and [10], and a more detailed performance evaluation is discussed in [11]. In short, the target and donor substrates are aligned and pressure is applied to form a gold-gold compression bond; the donor substrate is then removed, leaving the switches in place over the CPW lines [Fig. 1(c)].

The fabricated pull-down structures are shown in Fig. 2. In Fig. 2(a), the SEM micrograph shows the transferred relay on top of 


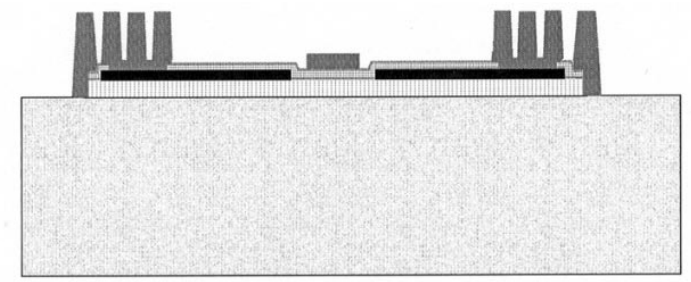

(a)

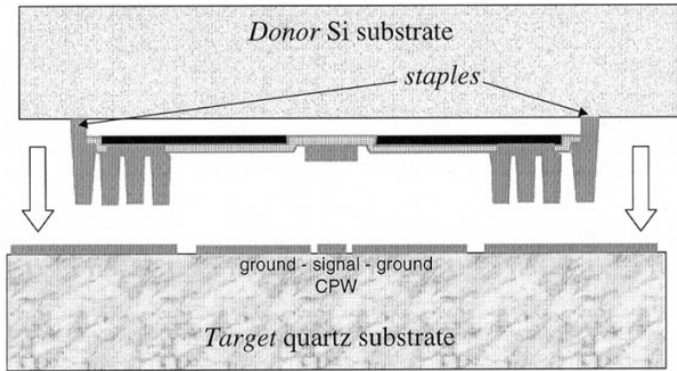

(b)

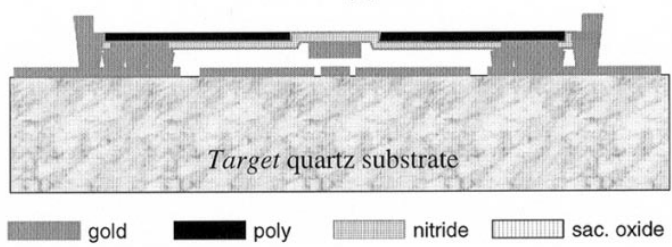

(c)

Fig. 1. Schematics of fabrication. (a) Example of structure before release and transfer. (b) Example of structure after release, flipped and aligned for transfer onto target substrate. (c) Final transferred structure.
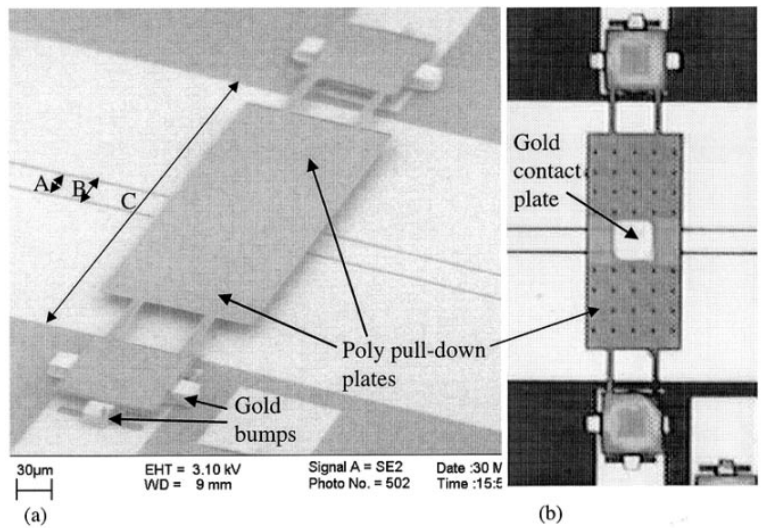

(b)

Fig. 2. Micrographs of electrostatic pull-down microwave switches batch transferred onto CPW transmission lines on a quartz substrate: (a) SEM of a typical device, and (b) an image of another device for series switching showing some misalignment in $x$ and in $y$. Dimensions $A=30 \mu \mathrm{m}, B=37 \mu \mathrm{m}$, and $C=420 \mu \mathrm{m}$. Each pull-down plate is $160 \mu \mathrm{m} \times 160 \mu \mathrm{m}$.

the CPW transmission line in ground-signal-ground (GSG) configuration. The individual parts of the relay may be better seen in the microphotograph in Fig. 2(b). The polysilicon pull-down plates extend from either anchored side over the ground planes of the CPW to maximize capacitance, thereby lowering actuation voltage. Over the signal strip of the CPW, only structural nitride and the gold contact plate are seen, designed to minimize parasitic effect on the CPW. The gold plate, seen in Fig. 2(b) is isolated by structural nitride and can make contact to the CPW

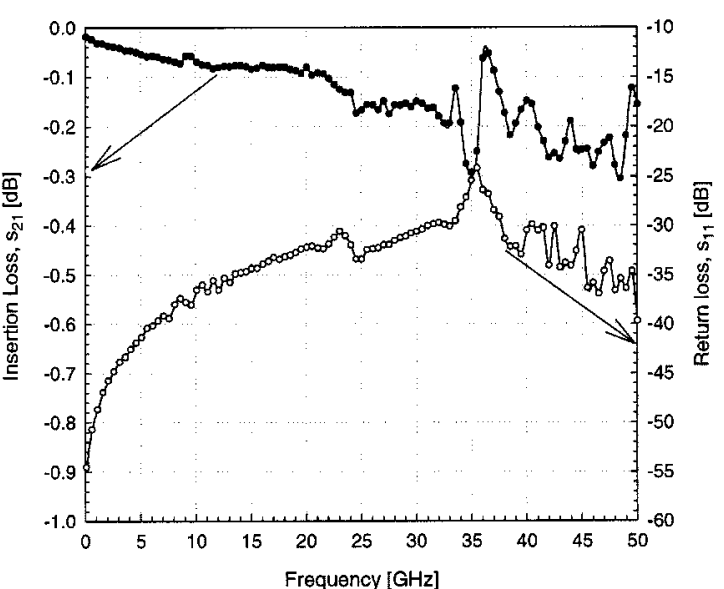

(a)

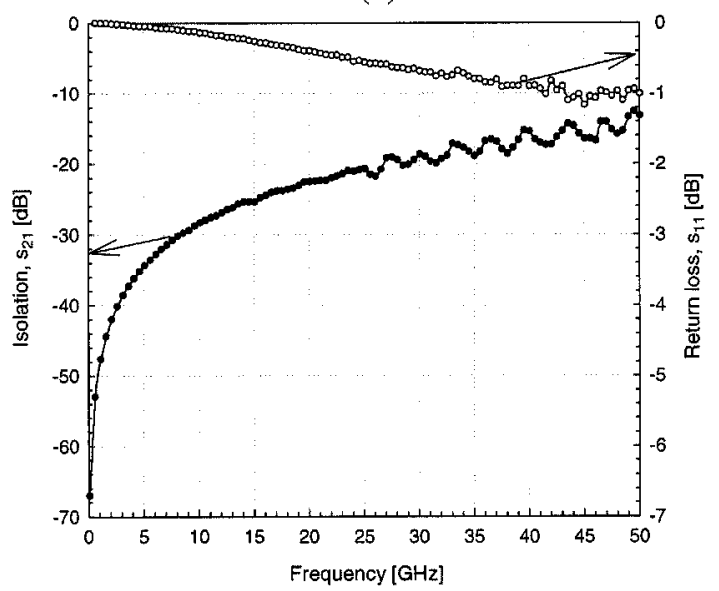

(b)

Fig. 3. Measured performance of a series pull-down microrelay: (a) DOWN-gold contact plate completing the CPW transmission line, and (b) isolation when UP.

below, either completing an open circuit in a series switch, or shorting the CPW strips in a shunt switch.

\section{DeVice Characterization}

\section{A. Low Frequency Testing}

The devices were tested by applying dc bias between actuating polysilicon plates (via pads) and CPW ground planes, and observing the actuation and subsequent change in resistance in the CPW. On one chip, simple pull-down structures exhibited turn-on voltages between 25 and $35 \mathrm{~V}$. On many other chips, however, pull-down voltages of 50-60 V were observed. Finally, there were cases of chips on which over $110 \mathrm{~V}$ had to be applied to fully actuate the structures. These large variations in the pull-down voltage are believed to be due to the chip-to-chip variation in the height of the structures from the target chip due to variations in flip-chip transfer. Most of the devices were tested with a current of $1 \mathrm{~mA}$ passing through the contact, and exhibited turn-on times on the order of $200 \mu \mathrm{s}$, and turn-off times below $20 \mu$ s. The switch of Fig. 2 was cycled over 200000 times before failing, but more study of reliability of the devices is required. In most cases, failure due to metal stiction was observed. 


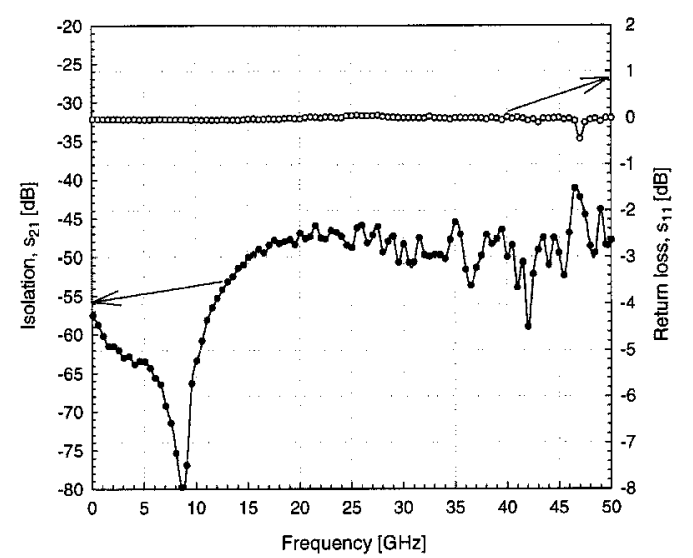

(a)

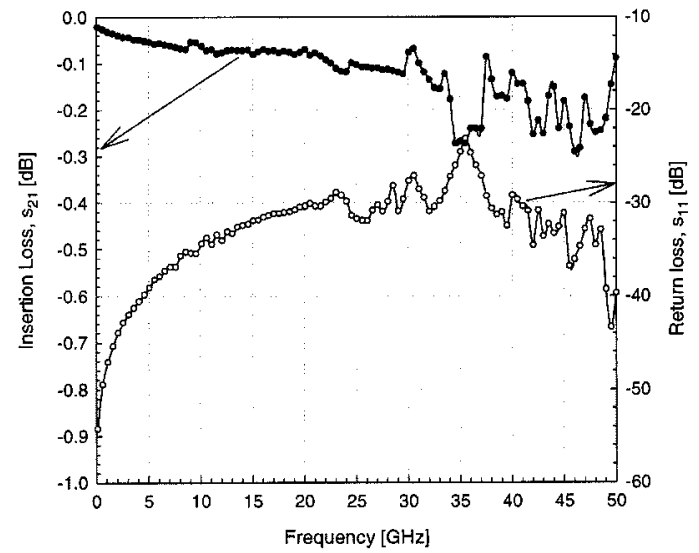

(b)

Fig. 4. Measured performance of a shunt pull-down microrelay: (a) DOWNisolation with gold contact plate shorting the CPW transmission line, and (b) insertion loss when UP.

\section{B. Microwave Testing}

Microwave testing was performed using a vector network analyzer and a probe station at frequencies from $100 \mathrm{MHz}$ to $50 \mathrm{GHz}$. Calibration and de-embedding was performed by measuring CPW transmission lines and reflects of different lengths on the same chip. After proper de-embedding [12], the measurement reference planes were brought to $0.050 \mathrm{~mm}$ before the microrelays on the test structure CPW. With the calibration, we measured the s-parameters while switching the devices off and on.

Firstly, we characterized the series pull-down switches [Fig. 2(b)] in which the gold contact plate completes the interrupted CPW signal strip when the switch is ON/DOWN. Fig. 3(a) shows the insertion loss and return loss in the ON state, and Fig. 3(b) shows the same parameters in the OFF/UP state. In this relay, the CPW gap was $60 \mu \mathrm{m}$, the structure was approximately $5 \mu \mathrm{m}$ above the CPW, and the contact plate overlapped the CPW signal line by $25 \mathrm{um}$ on each side. This resulted in isolation [Fig. 3(b)] of better than $20 \mathrm{~dB}$ up to $27 \mathrm{GHz}$, and better than $14 \mathrm{~dB}$ throughout the measured frequency range. Further, we tested shunt-type switches in which the gold contact plate shorts the CPW when the switch is down, resulting in isolation when actuated. Measured insertion loss and isolation of one such device is shown in Fig. 4. In the OFF/UP state, very low insertion loss is seen due to the uninterrupted CPW in this type of switch. In the ON/DOWN state the isolation was measured to be $>40 \mathrm{~dB}$ due to the relay's $30 \mu \mathrm{m}$ wide shorting contact plate with $12 \mu \mathrm{m}$ overlap over each ground plane.

\section{CONCLUSION}

We presented the design, fabrication, and batch-transfer integration of low-loss microrelays for microwave applications. Different types of switches were experimentally demonstrated, all based on polysilicon pull-down plates, structural/isolation nitride, gold bumps and gold-to-gold contacts. Their operation was characterized up to $50 \mathrm{GHz}$. A future goal is to extend the range of characterization frequencies and determine the upper frequency limit of operation. Also, long-term reliability of the structures and gold-to-gold contacts will be studied thoroughly, including operation at a variety of power ranges. Future designs will focus on reducing actuation voltages, lowering contact resistances, and improving actuation voltage uniformity. The latter will be resolved by including small hard-stop structures to pre-determine the exact distance after bond compression.

\section{ACKNOWLEDGMENT}

The authors would like to thank G. Rebeiz, M. Ozgur, A. Singh, D. Teasdale, B. Warneke, and L. Muller for useful technical discussions. The authors would also like to thank B. Jacobs, T. Michno and O. Villavicencio of the SPAWARSYSCEN San Diego, where the measurements were performed.

\section{REFERENCES}

[1] C. T.-C. Nguyen, L. P. B. Katehi, and G. M. Rebeiz, "Micromachined devices for wireless communications," Proc. IEEE, vol. 86, no. 8, pp. 1756-68, Aug. 1998.

[2] L. P. B. Katehi, G. M. Rebeiz, and C. T.-C. Nguyen, "MEMS and Si-micromachined components for low-power, high-frequency communications systems," 1998 IEEE MTT-S Int. Microwave Symp. Dig., vol. 4, pp. 331-333, Jun. 1998.

[3] Z. J. Yao, S. Chen, S. Eshelman, D. Denniston, and C. Goldsmith, "Micromachined low-loss microwave switches," J. Microelectromech. Syst., vol. 8, no. 2, pp. 129-34, June 1999.

[4] D. Hyman, A. Schmitz, B. Warneke, T. Y. Hsu, J. Lam, J. Brown, J. Schaffner, A. Walston, R. Y. Loo, G. L. Tangonan, M. Mehregany, and J. Lee, "GaAs-compatible surface-micromachined RF MEMS switches," Electron. Lett., vol. 4, pp. 1507-10, June 1999.

[5] J. B. Muldavin and G. M. Rebeiz, "30 GHz tuned MEMS switches," 1999 IEEE MTT-S Int. Microwave Symp. Dig., vol. 4, pp. 1511-1514, June 1999.

[6] K. Suzuki, S. Chen, T. Marumoto, Y. Ara, and R. Iwata, "A micromachined RF microswitch applicable to phased-array antennas," 1999 IEEE MTT-S Int. Microwave Symp. Dig., vol. 4, pp. 1923-1926, June 1999.

[7] K. F. Harsh, W. Zhang, V. M. Bright, and Y. C. Lee, "Flip-chip assembly for Si-based RF MEMS," in Proc. 12th IEEE Int. Conf. Microelectromechanical Systems (MEMS '99), Orlando, FL, Jan. 1999, pp. 273-278.

[8] Z. Feng, W. Zhang, B. Su, K. F. Harsh, K. C. Gupta, V. Bright, and Y. C. Lee, "Design and modeling of RF MEMS tunable capacitors using electro-thermal actuators," 1999 IEEE MTT-S Int. Microwave Symp. Dig., vol. 4, pp. 1507-10, June 1999.

[9] M. M. Maharbiz, R. T. Howe, and K. S. J. Pister, "Batch transfer assembly of micro-components on surface and SOI MEMS," in Transducers '99, Sendai, Japan, June 7-10, 1999, pp. 1478-81.

[10] M. M. Maharbiz, M. B. Cohn, R. T. Howe, R. Horowitz, and A. P. Pisano, "Batch micropackaging by compression-bonded wafer-wafer transfer," in MEMS '99, Orlando, FL, Jan. 17-21, 1999, pp. 482-9.

[11] A. Singh, D. Bilic, and R. T. Howe, "Performance evaluation of batch-transferred surface micromachined resonators," in Transducers '99, Sendai, Japan, June 7-10, 1999, p. 1158.

[12] R. B. Marks, "A multiline method of network analyzer calibration," IEEE Trans. Microwave Theory Tech., vol. 39, pp. 1205-1215, July 1991. 